\title{
Resolution Enhancement at Low-Accelerating-Voltage by Improvements of Diffraction Limit and Chromatic Aberration
}

\author{
H. Sawada ${ }^{1}$, T. Sasaki ${ }^{1}$, F. Hosokawa ${ }^{1}$, and K. Suenaga ${ }^{2}$ \\ ${ }^{1}$ JEOL Ltd., 3-1-2 Musashino, Akishima, Tokyo, 196-8558, Japan \\ ${ }^{2}$ National Institute of Advanced Industrial Science and Technology (AIST), Tsukuba, 305-8565, Japan
}

An atomic-resolution microscope operating at a lower voltage is increasingly requested because the lower accelerating voltage provides less specimen damage and larger scattering cross-section that results in a high signal for analysis [1-3]. However, a resolution reduction by a diffraction limit becomes severe with an increase in the wavelength of an electron as an accelerating voltage decreases. Another resolution reduction is caused by a chromatic aberration due to an energy spread of the electron source. For maintaining atomic resolution at low accelerating voltage, a larger convergence angle realized with an aberration corrector and a narrower energy spread of the source are required. The developed microscope by us achieved atomic resolution at the accelerating voltage lower than $60 \mathrm{kV}$, using a higher-order aberration corrector [4]. The atomic-resolution imaging showing $136 \mathrm{pm}$ resolution using a Si [110] has been demonstrated at 30 and $60 \mathrm{kV}$ [5]. This paper reports the realization of sub-angstrom imaging at $60 \mathrm{kV}$ using a Ge [112] specimen which shows 82-pm dumbbells [6].

The developed microscope was equipped with a CFEG. The electron source for the gun was chosen to be a tungsten [310] tip to obtain a narrow energy spread. In our observation, the energy spread was measured to be $0.36 \mathrm{eV}$ in full width of half maximum (FWHM), after setting of an extracting voltage to provide a proper emission current used for a high resolution imaging. A demagnification factor of probe on a specimen refer to the source in probe forming lens system was set so as to obtain a Gaussian probe size of $14 \mathrm{pm}$ on a specimen. Figure 1(a) shows the calculated probe profiles at convergence semi-angles of $30 \mathrm{mrad}, 35 \mathrm{mrad}, 40 \mathrm{mrad}$, and $45 \mathrm{mrad}$. The probe profiles show sharper peaks with an increase in the convergence semi-angle. The probe profile at $45 \mathrm{mrad}$ shows diameters of $57.8 \mathrm{pm}$ in FWHM, $91.4 \mathrm{pm}$ in D50 and $120.4 \mathrm{pm}$ in D59, where D50 and D59 denote the diameter of the electron beam that include $50 \%$ and $59 \%$ of the total beam current, respectively. The probe diameter at $45 \mathrm{mrad}$ expected to realize sub-angstrom STEM imaging at low accelerating voltages. Figure 2 shows a set of simulated STEM images of Ge [112] at $60 \mathrm{kV}$ at several convergence semi-angles [7]. The dips between the atomic dumbbells become deeper with an increase of convergence angle. The large diffraction limit, due to a longer wavelength at a low accelerating voltage, worsens the resolution in the sub-angstrom imaging. Judging from the simulations, at a convergence semi-angle larger than $40 \mathrm{mrad}$, the 82-pm dumbbell is expected to be resolved because of the small diffraction limit.

For experimental evaluation for the sub-angstrom resolution, a Ge [112] crystalline specimen was observed at $60 \mathrm{kV}$, as shown in Fig. 3. The current and convergent semi-angle for the probe were measured to be $6 \mathrm{pA}$ and $45 \mathrm{mrad}$. The simulated image at $45 \mathrm{mrad}$ is displayed at the upper left of Fig. 3(a). Figure 3(b) shows a Fourier transform of the STEM image shown in Fig. 3(a). The Ge-Ge dumbbells are resolved in Fig. 2(a), and the $44 \overline{4}$ spot (corresponding to $(82 \mathrm{pm})^{-1}$ ) is clearly visible in Fig. 3(b). 
We achieved a resolution of $82 \mathrm{pm}$ using the Ge-Ge dumbbell structure by high angle annular dark-field imaging. We also experimentally confirmed that resolution at a low accelerating voltage is severely affected by a convergence angle and a energy spread of the probe [8]. This work was supported by JST under the Research Acceleration Program (2012-2016).

\section{References:}

[1] K. Suenaga, and M. Koshino, Nature 468 (2010), p. 1088.

[2] O. L. Krivanek, et al, Nature 464 (2010), p. 571.

[3] Z. Lee, et al, Ultramiscoscopy 112 (2012), p. 39.

[4] H Sawada et al, J. Electron Microsc. 58, (2009), p. 357.

[5] T. Sasaki, et al, J. Electron Microsc. 59 (2010), p. S7.

[6] M. O'Keefe et al, J. Electron Microsc. 54 (2005), p. 169

[7] F. Hosokawa et al, unpublished (2013).

[8] H. Sawada et al, Micron (2013) DOI : 10.1016/j.micron.2014.01.007.

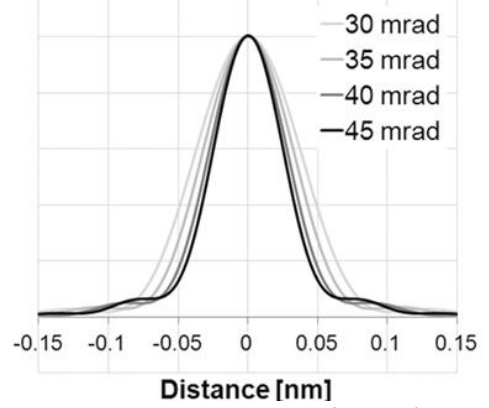

Figure 1. (a) Calculated probe shapes at convergence semi-angles of $30 \mathrm{mrad}, 35 \mathrm{mrad}, 40 \mathrm{mrad}$, and $45 \mathrm{mrad}$.

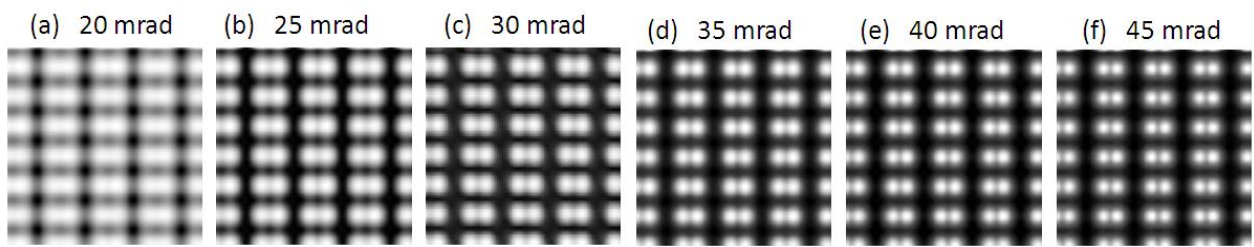

Figure 2. Simulated dark-field images of the Ge [112] specimen at convergence semi-angles of (a) 20 mrad, (b) $25 \mathrm{mrad}$, (c) $30 \mathrm{mrad}$, (d) $35 \mathrm{mrad}$, (e) $40 \mathrm{mrad}$, and (f) $45 \mathrm{mrad}$ at $60 \mathrm{kV}$ [7] by the multi-slice method.

\section{(a)}

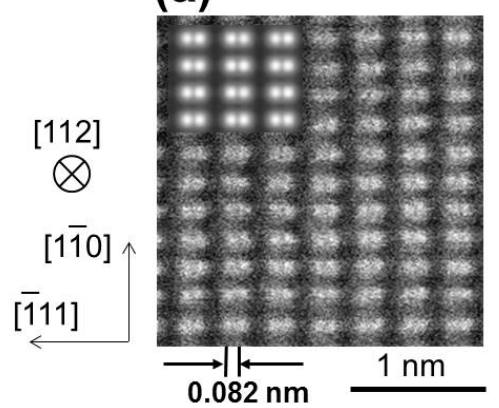

(b)

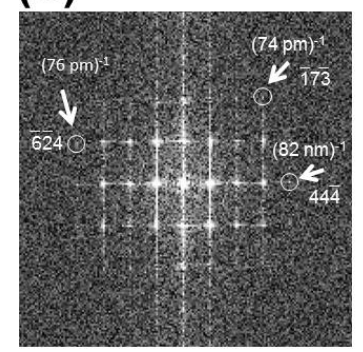

Figure 3. (a) The Ge [112] dark-field image at $60 \mathrm{kV}$. (b) The power spectrum of Fourier transform for the STEM image in (a). The pattern clearly shows spots of $(82 \mathrm{pm})^{-1}$. 